\title{
The Design of The Positioning Mechanism of The Mould Car in The Intelligent Manufacturing Production Line of Sanitary Ceramics
}

\author{
Bo Zhong ${ }^{1 *}$, Huachao Lv ${ }^{1}$, Hui Gao ${ }^{1}$, Ying Wang ${ }^{1}$ \\ ${ }^{1}$ China building materials inspection and Certification Group Shaanxi Co., Ltd. Xi'an 710116, Shaanxi
}

\begin{abstract}
The social production demand of intelligent manufacturing production line of sanitary ceramics was expounded, the technological process of intelligent manufacturing of sanitary ceramics was analyzed, and the importance of mold car positioning in the whole system was put forward. This paper analyzes and explains the positioning mechanism, introduces its structural features, functions and characteristics, and explains its working principle in detail. It proves the superiority of intelligent manufacturing production line of sanitary ceramics, which has certain reference value and guiding significance for the manufacture and production of sanitary ceramics in China.
\end{abstract}

\section{Introduction}

With the rapid development of science and technology, the high speed and automation of the production process of industrial and civil products have been paid more and more attention by various enterprises. The automation of sanitary ceramics started late in China. Since then, the production of sanitary ceramics has been mainly based on labor, with high labor intensity, low efficiency, high production cost and low yield, which has greatly weakened the competitiveness of the market. Intelligent manufacturing production line, therefore, development of sanitary ceramics for ceramic production enterprises to provide more energy saving, environmental protection, more efficient machinery and equipment, reduce the labor intensity, improve work efficiency, reduce production costs, increase productivity, save energy, is of great significance for China's ceramic industry, but also brings huge economic benefits to the society. Grouting circulation line is an important part of intelligent manufacturing production line of sanitary ceramics. So the positioning mechanism of the mold car has become an important part of the whole production line. ${ }^{[1]}$

\section{Analysis of Grouting Circulation Line Process Flow}

This paper takes the conjoined toilet as an example to illustrate the grouting circulation line. The equipment can realize that the overall size difference is not big, and the grouting process of the conjoined toilet with different shapes remains unchanged. The process flow of grouting circulation line is shown in Figure 1. 


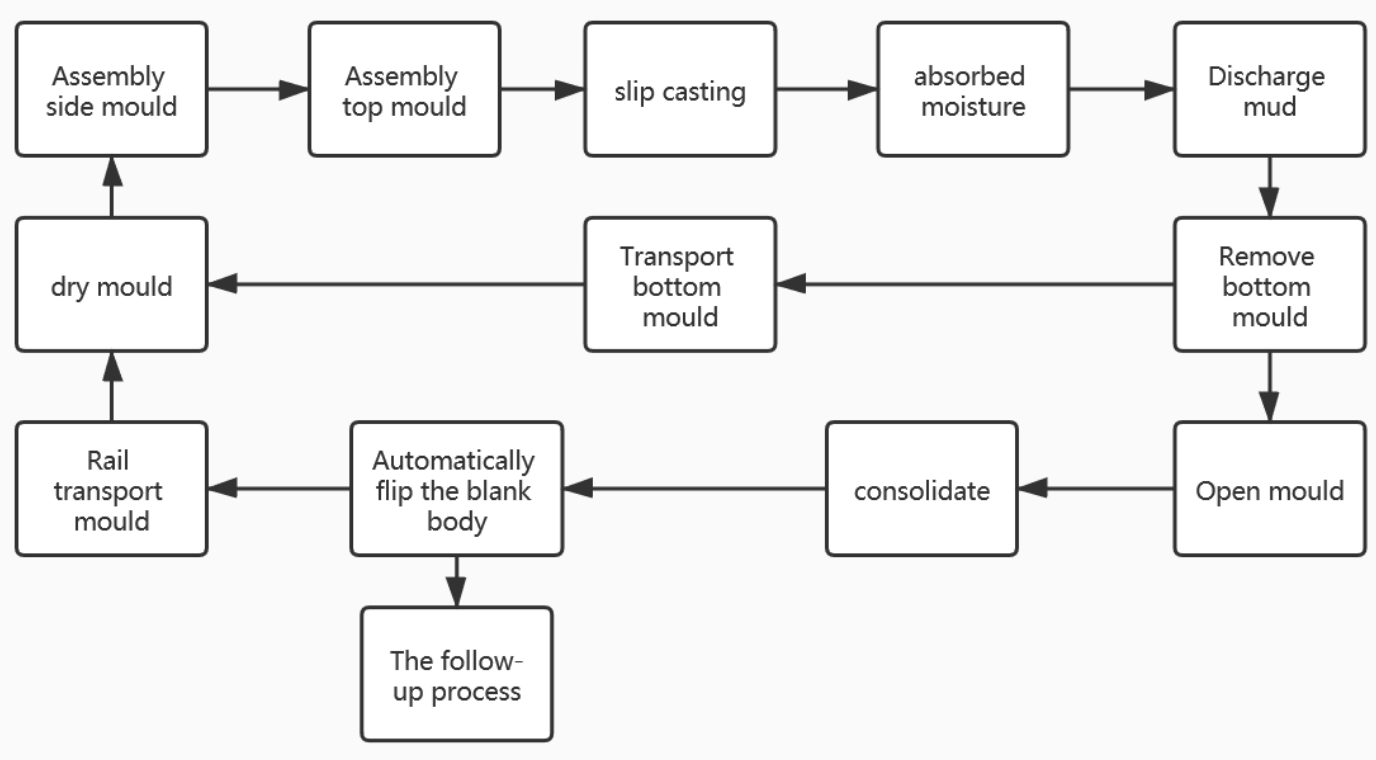

Fig 1. Process Flow Chart of Grouting Circulation Line

The whole production process is as follows:

a) The starting point of this paper is the clamping position. After the dry mold and the mold trolley are transported to the mold closing position through the ground circulation line. The top die and the supporting plate carrying the top die are transported to the closing position via an air double speed chain.

b) The clamping machine shall tighten the clamping of the open side of the mold car. Then the clamping machine grabs the bottom die on the double speed chain and places it in the corresponding position. Finally, the worker will quickly lock up.

c) The mold car is transferred to the grouting area through the ground circulation line. When the mold trolley for the whole production line is in place, the worker inserts the grouting pipe and micro-pressure pipe into the mold trolley. After grouting, slurry suction, slurry release and mold opening process, the mold car will be transferred to the position of the billet machine.

d) After opening the mold, the bottom mold is once again grabbed onto the bottom mold support plate on the air multi-speed chain. Then transfer to the drying room, waiting for the next closing mold.

e) The mold trolley arrives at the blank turning position. After positioning, the die tray is fed by the billet receiving machine and fastened vertically to the open die trolley. Then the billet turning machine is opened, and the linkage with the billet receiving machine makes the whole billet turning $90^{\circ}$ Angle. At this point the blank body has been placed horizontally on the mold tray.

f) After that, the blank body is transferred to the subsequent process position through the ground doublespeed chain. The mold car continues to flow into the drying room, waiting for the next closing of the mold.

\section{System Structure}

\subsection{Mould trolley structure}

The mould is composed of top mold, side mold, bottom mold, rear body mold at the position of drain pipe, small pieces in the water tank, and hanging pieces in the urinal.

The die trolley is composed of a base, side bracket and bottom bracket welded by a square pipe of 50 as the main body, and a bottom die mounting seat consisting of a rectangular pipe of $50 \times 25$ as the main body. The side bracket and the base are connected by a linear slide rail and slider, and the side bracket can slide freely on the base. The bottom bracket is linked with the base by hinge. When the bottom bracket is turned over, the turning machine can drive the bottom bracket to complete the turning process. The bottom film mounting bracket and side bracket are locked with wedge block, which is convenient to realize automatic extraction of the bottom die when opening and automatic locking when closing the die. Six track wheels are installed on the base of the trolley, which can make the trolley travel in a straight line on the light rail. Four locking buckles were added on both sides of the trolley, which were manually locked after mold closing to ensure the mold was fully fitted and prevent slurry leakage during grouting. ${ }^{[2]}$

\subsection{Running crawler structure}

The running crawler provides accurate guide rail mechanism for the running of the die trolley. It provides traction power and positioning block structure for the start and stop of the die trolley, and the traction power runs intermittently back and forth to ensure the traction distance is accurate and stable. The crawler has good adjustment performance, which ensures the adjustable height and consistent level of the guide rail mechanism in 
the running of the die trolley. There is a unique tilting mechanism on the running crawler, which ensures that the mold can release the pulp quickly and leave no residual pulp in the process of releasing the pulp.

\section{Description of Positioning Mechanism Structure}

Through the description of the whole production process, it can be seen that the precise positioning of the mold car is mainly in the three links of closing the mold, opening the mold and turning over the blank. Because the die opening and - die process involves the butt joint of the die and the die car. The billet turning process involves the butt joint between the die tray and the billet body.

Through the structure of the die car, it is not difficult to see that the overall load of the die car is too large. Therefore, it is necessary to reduce the height of the mold car from the ground as far as possible in the process of running the mold car to ensure the stable and safe running of the car. By running the crawl line structure, it can be seen that the internal structure of the line body is complex, including the motor, decoupling and other large structures. So the size of the positioning mechanism becomes particularly important.

The main structure and process flow of the positioning mechanism:

The main structure of the positioning mechanism is shown in Figure 2 and Figure 3

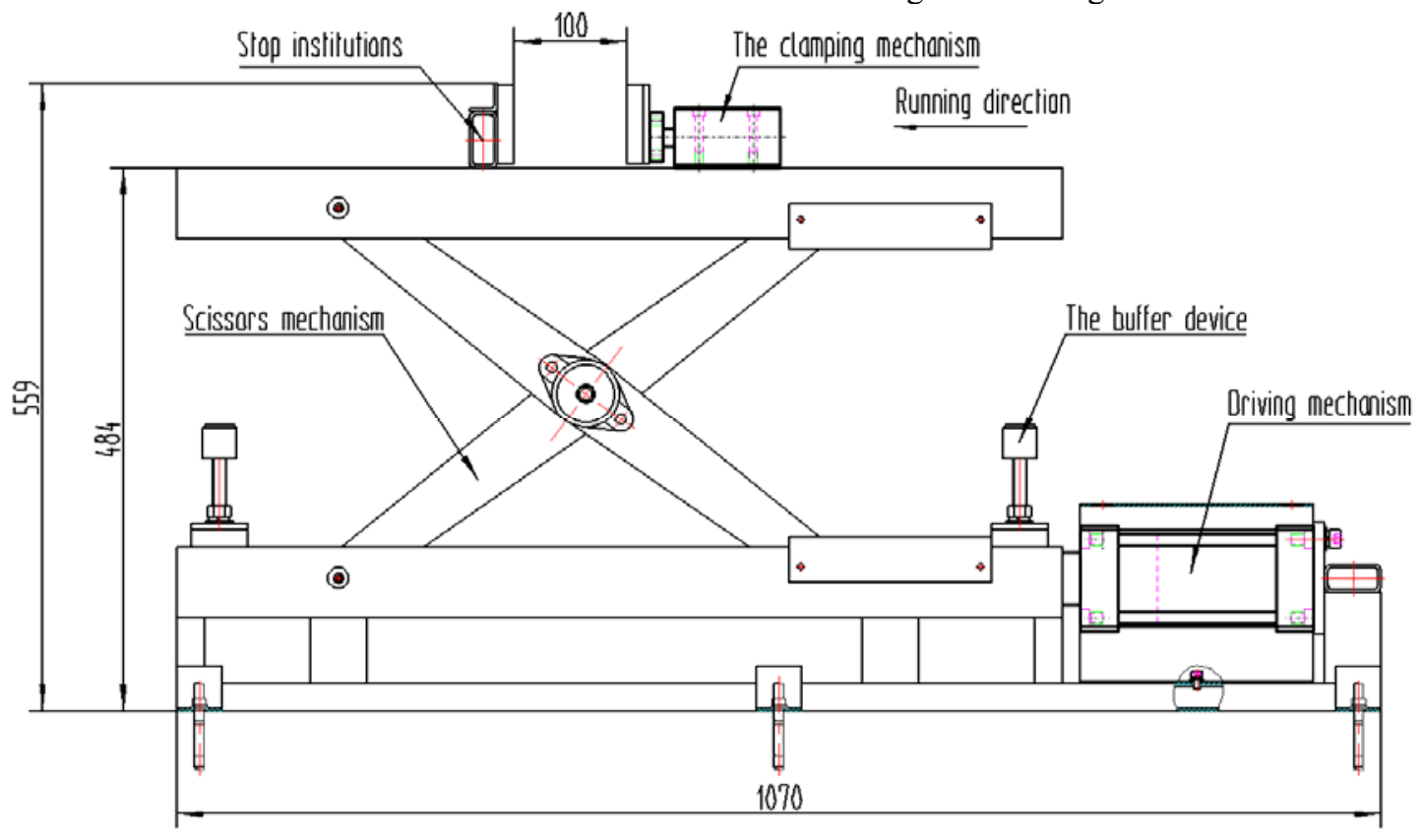

Fig 2. Lifting structure diagram of positioning mechanism

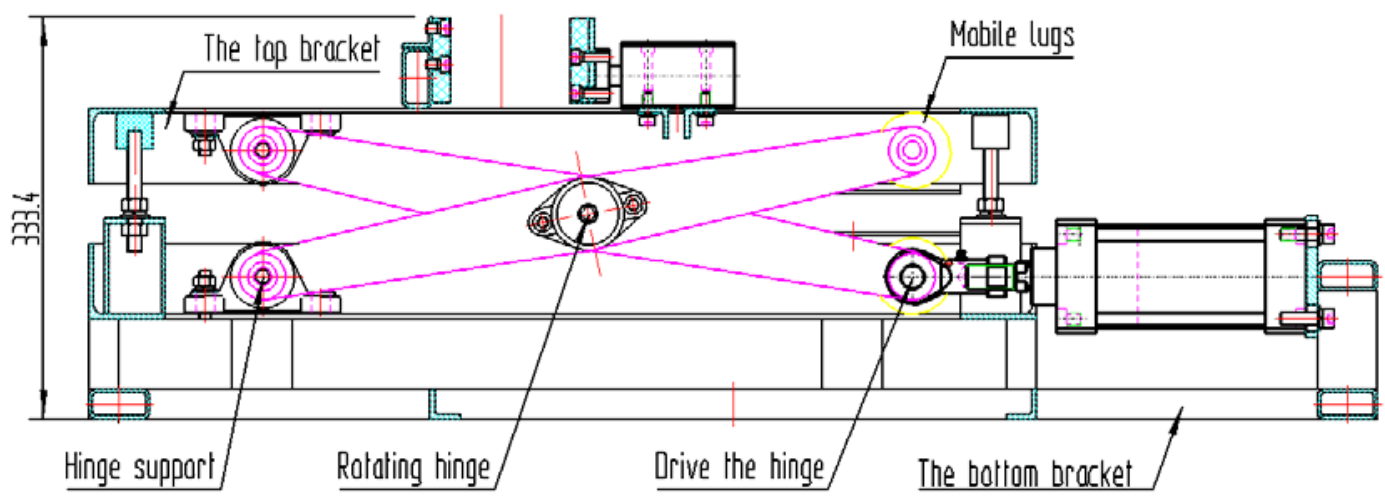

Fig 3. Structure diagram of positioning mechanism descending

Positioning process flow:

a) After the mold trolley reaches the specified position, the driving mechanism pushes forward.

b) The drive hinge drives the caster to migrate, resulting in the rotation of the hinge.

c) At this point, under the action of the supporting hinge, the overall Angle of the cutting knife and fork mechanism is reduced to drive the top platform to lift.

d) The clamping mechanism and blocking mechanism will be located on both sides of the positioning beam at the bottom of the mold car.

e) At this time, the clamping mechanism drives 
the mold car towards the blocking mechanism, and the mold car is positioned and clamped.

f)After the operation of the mold car is completed, the clamping mechanism will unload and loosen the mold car.

g) The driving mechanism moves in the opposite direction, so that the overall Angle of the cutting knife and fork mechanism is enlarged and the top platform is driven down.

h) The top platform falls onto the cushion at the bottom of the platform, and then the mold car flows away.

\section{Structural advantages:}

a) Due to the heavy weight of the mold car, it is inevitable that the mold car will continue to move forward due to inertia in the process of movement. But the distance that each mold car moves because of inertia is different again. So the distance between the blocking mechanism and the clamping mechanism is increased. And the movement direction of the clamping mechanism is the same as that of the die trolley, which avoids the inaccurate positioning caused by the machining error of the die trolley.

b) In order to ensure the safety of personnel, the overall height of the running crawler is too low, so that the purpose of vertical drive jacking cannot be completed in the space. This mechanism adopts the cutting knife and fork mechanism, which greatly reduces the volume of the whole vertical direction through the way of horizontal driving. It effectively avoids the main mechanism in the crawl line.

\section{Conclusion}

Automated production lines can replace traditional production techniques and greatly reduce human labor. The positioning mechanism can precisely position the mold trolley, reduce the damage of the blank body in the production process, and thus ensure the quality of the blank body. Improved business and corporate earnings. It has certain reference value and guiding significance to the design and development of other types of sanitary ceramics machinery.

\section{Acknowledgments}

This work was supported by the study was supported by Public Service Platform Project of Industrial Technology Foundation of Ministry of Industry and Information Technology in 2020 (No.2020-0098-2-1)

\section{Reference}

1. Li Meichuan, Zhang Qi. Design and Research of Intelligent Manufacturing Line for Sanitary Ceramic $[\mathrm{J}]$. China Building Materials Science \& Technology,2018:71-75

2. Zhang Qi, Li Meichuan. Technology and Design of Intelligent Casting and Grouting Circulation Line for Sanitary Ceramics $[\mathrm{J}]$. China Building Materials Science \& Technology,2018:82-84

3. Bai Zhanying, Zhang Weixing, Liu Xiaoyun.
Technical status and development trend of building and sanitary ceramics industry in China [J]. Ceramics, 2011 (12): 40-43

4. Li zewen. Development status and Prospect of domestic ceramic industry $[\mathrm{J}]$. Foshan ceramics, 2011,21 (09): 6-11

5. Nie Baomin. Development and direction of China's sanitary ceramics industry [J]. Ceramics, 2011 (04): 21-24

6. Li Jiyuan, Ma Yushu. Comprehensive experimental design of ceramic grouting molding $[\mathrm{J}]$. Science and technology innovation guide, 2013 (09): $247+249$

7. Deng Zhuming, Gou Li, ran Junguo. Rheological properties of Yb: YAG laser ceramic slurry prepared by grouting [J]. Silicate bulletin, 2013,32 (02): 221$224+230$

8. Li Jia. Study on the performance of plaster mold for sanitary ceramics by grouting [D]. South China University of technology, 2012 